\title{
A Method for Blink Artifact Detection and Removal with Wavelet Transform and Hilbert Transform
}

\author{
Xiaobai CAI* \\ China Rehabilitation Research Center \\ School of Rehabilitation Medicine \\ Capital Medical University \\ Beijing, China \\ e-mail: caixb@bit.edu.cn
}

\author{
Junjun CHEN \\ Second Clinical Hospital \\ Jilin University \\ Changchun, China \\ e-mail: Chenjj-1218@163.com
}

\begin{abstract}
This paper presents a method of blink artifact removal of single lead EEG signal. After a wavelet transform signal singularity points are detected and modulus maxima values of wavelet transform are applied to find peaks of blink artifacts. By using Hilbert transform instantaneous frequency is obtained. With the peaks as clues instantaneous frequencies are used to identify the zones appearing blink artifacts. With a stationary wavelet transform, EEG signal is decomposed to the depth on which the frequency bandwidth of approximation coefficients covers the frequency bandwidth of blink artifacts. The approximation coefficients in a blink artifact zone are estimated by the fitting data using the data outside of the blink zone. To the detail coefficients de-noising threshold value in a blink zone is estimated with the threshold values of two sides out of the blink zone. After these coefficients are corrected, the signal is reconstructed to get the EEG signal with blink artifact removal. The experimental results show that the proposed method is effective to detect and remove blink artifacts and avoid affecting the EEG signal outside the blink artifact zones.
\end{abstract}

Keywords- EEG; blink artifact; wavelet transform; Lipschitz index; Hilbert transform; blink zone

\section{INTRODUCTION}

In brain science and neuroscience electroencephalogram (EEG) is a noninvasive biomedical signal for researching and recognizing normal brain activity and abnormality. However, the weak signal from the scalp is mixed with some interference that makes the interpretation of EEG signal difficult, in which physiological artifacts are major sources. The artifacts include eye blinking and movement, heart beating and muscle movement. Among them blink artifact has considerable influence to the analysis of EEG signals due to larger amplitude, higher occurrence rate, so that blink artifact removal is a concerned problem [1-2].

Due to the ability of wavelet transform in time frequency analysis, some researchers use it in the problem of detection and removal of blink artifact in EEG signals. For the processing of single lead EEG signal, Krishnaveni[3] presents a method to identify the artifact zones of blink and eye movement and apply an adaptive threshold value algorithm only to the identified zones. The approximation coefficients at button level are reconstructed. The reconstructed signal sample points are compared with its successive sample points to identify the edges classified, depending on whether the edges correspond to blinks or not correspond to blinks, based on their relative amplitude. The identified edges are scaled to four integers and summed up getting the edges information in a single array. The later is traversed to identify unique patterns of the artifact zones. Since a blink artifact is not a real step-function signal, this method is difficult to determine the zones that don't have steep edges. M. Kaiyu [4] proposes a method of blink artifact removal based on singularity detection and threshold de-noising. In the method wavelet is decomposed by a cubic B-spline wavelet. The Lipschitz index is used for detecting blink artifact peaks. For the estimation of a blink artifact zone an empirical statistical value is used, by which the width of the zone is set to 0.5 seconds. The empirical statistical value based on biomedicine is conservative. Kumar [5], proposes a statistical method for removing blink artifact from EEG recordings through wavelet transform without using an electrooculogram reference channel. In the method, the detail coefficients of wavelet decomposition are used. Each three coefficients are compared sequentially to identify the spikes in the EEG signal at each level, and then to identify the ocular artifact spike zones using coefficient of variation. An empirical threshold value and a threshold function are given and implemented on obtained zones to process coefficients.

This paper presents a method of blink artifact removal. At first based on wavelet analysis signal singularity points are detected and modulus maxima values of wavelet transform are utilized, to find peaks of blink artifacts. Next with the peaks as clues, instantaneous frequencies, obtained by Hilbert transform, are used to find the zones appearing blink artifacts. Then, based on a stationary wavelet transform, the approximation coefficients of a blink artifact zone are estimated by the nearby data out of the zone, and the detail coefficients are processed with the threshold values estimated from the non blink zones for high frequency de-noising. At last the signal is reconstructed to obtain the EEG signal with blink artifact removal. 


\section{The DETECTION OF BLINK ARTIFACTS}

\section{A. The Peak Detection of Blink Artifacts}

EEG signals, carrying information about rhythmic activities at different frequency bandwidths of $\delta(0.5-4 \mathrm{~Hz})$, $\theta(4-8 \mathrm{~Hz}), \alpha(8-13 \mathrm{~Hz}), \beta(13-30 \mathrm{~Hz})$ and $\gamma(30-50 \mathrm{~Hz})[6]$, are recorded using electrodes placed across the scalp. EEG waveforms are characterized by three components, including amplitude, frequency and shape. Eye blinking generates spike-like shaped signal waveforms in a very short period of 200-400 ms and low frequency bands such as $\delta$ and $\theta$ [7]. Fig. 1 is a section of the EEG signal waveform that has two blink artifacts.

The spike-like shaped signal waveforms, suddenly and stochastically appearing, indicate existing singularities. Singularities often carry the most useful information in signals, which are given by transient phenomena such as peaks. In bio-physics, it is also important to study the singularities to infer properties about the bio-physical phenomena [8]. In mathematics singularity can be quantitatively described by Lipschitz index. Lipschitz index is defined as follows: if there are a constant $M>0$ and $n=[\alpha]$ order polynomial $p_{t_{0}}(t)$, and a mathematic relation:

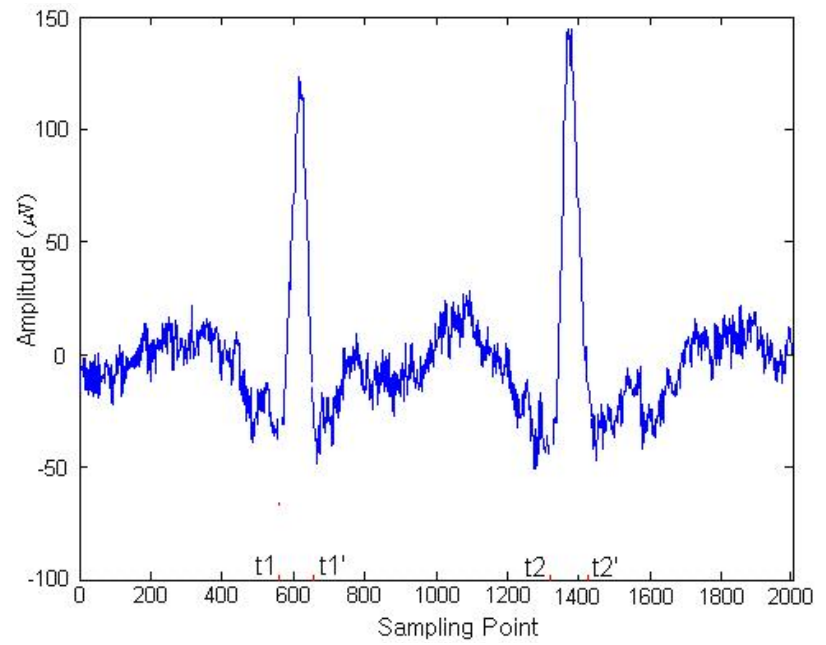

Figure 1.The waveform of the EEG signal with blink artifacts.

$$
\left|x(t)-p_{t_{0}}(t) \leq M\right| t-\left.t_{0}\right|^{\alpha}, \forall t
$$

then $x(t)$ is called as having a Lipschitz index $\alpha$ at the point $\mathrm{t}_{0}$. If $x(t)$ at the $\mathrm{t}_{0}$ is $\mathrm{n}$ times differentiable, but the $n$ order derivative is not continuous, then it is $n+1$ times not differentiable. In this time at the $\mathrm{t}_{0}$ point $x(t)$ has the Lipschitz index $\alpha$ and $n \leq \alpha<n+1$. Thus it can be seen that Lipschitz index shows the irregular degree of signal $x(t)$ at the $t_{0}$ or the interval $[a, b]$ around the point $t_{0}$. The more smooth $x(t)$ at here, the larger the corresponding $\alpha$. If $\alpha<1$, it is called that the signal exists a singularity at this point.

On the other hand, there is a close relation between Lipschitz index and wavelet transform of a signal. For a signal $x(t) \in \mathrm{L}^{2}(\mathrm{R}), \mathrm{x}(\mathrm{t})$ with Lipschitz index $\alpha \leq \mathrm{n}, \mathrm{n} \in$ $\mathrm{Z}^{+}$the order of vanishing moment of wavelet $\Psi(t)$, there exists a constant $\mathrm{A}$ that has the following formula:

$$
\left|\mathrm{WT}_{\mathrm{x}}(\mathrm{a}, \mathrm{b})\right| \leq \mathrm{Aa}^{\alpha+0.5}
$$

In the formula (2) A is constant, $\left|\mathrm{WT}_{\mathrm{x}}(\mathrm{a}, \mathrm{t})\right|$ is called as wavelet transform modulus of the signal $x(t)$. If there is a point $\left(\mathrm{a}_{0}, \mathrm{t}_{0}\right)$ that has the following relationship:

$$
\frac{\partial \mathrm{WT}_{x}\left(\mathrm{a}_{0}, \mathrm{t}_{0}\right)}{\partial \mathrm{t}}=0
$$

Then $\left(\mathrm{a}_{0}, \mathrm{t}_{0}\right)$ is the local extreme position of $\mathrm{WT}_{\mathrm{x}}(\mathrm{a}, \mathrm{t})$. When $t$ is located in the left neighborhood or the right neighborhood of $\mathrm{t}_{0}$ and $\left|\mathrm{WT}_{\mathrm{x}}(\mathrm{a}, \mathrm{t})\right| \leq\left|\mathrm{WT}_{\mathrm{x}}\left(\mathrm{a}_{0}, \mathrm{t}_{0}\right)\right|$ is satisfied, $\left(\mathrm{a}_{0}, \mathrm{t}_{0}\right)$ is called as modulus maximum point. We use a dyadic wavelet transform and let the scale of wavelet transform $a=2^{j}$, and on both sides of the formula (2) take a logarithm by 2 as the base of the logarithm. The formula (4) is gotten.

$$
\log _{2}\left|\mathrm{WT}_{\mathrm{x}}(\mathrm{a}, \mathrm{b})\right| \leq \log _{2} \mathrm{~A}+j(\alpha+0.5)
$$

With the formula the scale features of wavelet transform $\mathrm{j}$ and Lipschitz index $\alpha$ are connected. And the index $\alpha$ can be calculated based on the formula.

The clean EEG signal is similar to white noise, with the Lipschitz index $\sigma=-1 / 2-\varepsilon<0$. The wavelet transform coefficients quickly reduce with the increase of wavelet scale j. However to the Lipschitz index of singularity points, $0<\alpha<1$, at the modulus maximum point, the wavelet transform coefficient values increase with the increase of wavelet scale j. According to these principles, we design an algorithm and implement a computer program to detect out the peaks of blink signals.

\section{B. The Zone Identification of Blink Artifacts}

The peaks of blink artifacts tell us the information about blink appearances and its locations. But it is more important to identify the zones of blink appearances, which is a difficult problem [3-5]. To the problem, we notice the amplitude and frequency characteristics of blink artifacts, and utilize Hilbert transform to calculate instantaneous amplitude, phase and frequency. The instantaneous frequencies obtained are used to identify the zones of blink appearances.

Hilbert transform [9] represents a real signal to a complex signal. If a real signal is set as $x(t), \hat{x}(t)$ can be obtained by Hilbert transform. The $x(t)$ and $\hat{x}(t)$ form a complex conjugate pairs. They constitute an analytic signal as the following expression: 


$$
\bar{x}(t)=x(t)+j \hat{x}(t)=\mathrm{a}(t) e^{j \theta(t)}
$$

In the equation, $x(t)$ is the real part of $\bar{x}(t)$, a real channel recording. $\hat{x}(t)$ is the imaginary part of $\bar{x}(t)$, an orthogonal channel recording. In the equation, $a(t)$ is the instantaneous amplitude and $\theta(\mathrm{t})$ the instantaneous phase, and they have the following computing relationships:

$$
\begin{aligned}
& a(t)=\sqrt{x^{2}+\hat{x}^{2}(t)} \\
& \theta(t)=\arctan \frac{\hat{x}(t)}{x(t)}
\end{aligned}
$$

The instantaneous frequency can be calculated by the following equation:

$$
f(t)=\frac{1}{2 \pi} \frac{d \theta}{d t}
$$

From a purely theoretical point of view, this definition of instantaneous frequency may have a paradox. However because it has practical significance in explaining the characteristics of signals, as well as rationality and practicability, it is adopted in physics [9-11].

The clean EEG signal has characteristics of white noise. Since frequency bandwidth of blink artifact covers a small range of EEG signals in low frequency band and it's amplitude is significantly higher than that of clean EEG signal, in a zone of blink appearances clean EEG signal is masked by artifact signal. The domain components of the zones have smaller squared absolute values of instantaneous frequency such as the marked zones $\left[t_{1} \sim t_{1}^{\prime}\right]$ and $\left[t_{2} \sim t_{2}^{\prime}\right]$ in Fig. 2, in which $t_{1}, t_{1}^{\prime}, t_{2}$ and $t_{2}^{\prime}$ are correspond to the same positions in Fig. 1.

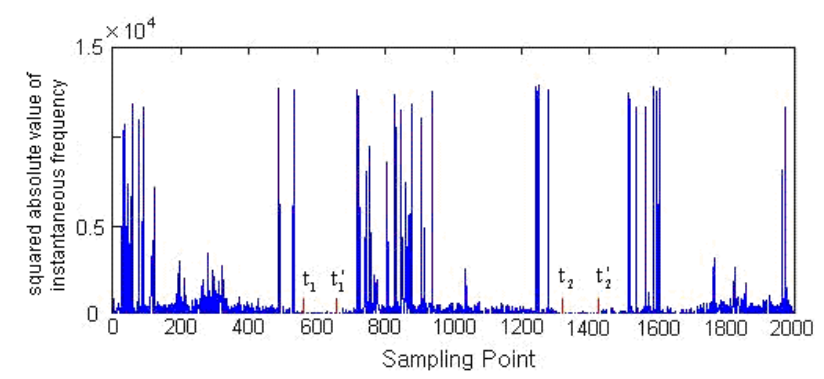

Figure 2. The squared absolute value of instantaneous frequency of the EEG signal with blink artifacts.

Based on this principle, we propose an algorithm to estimate the starting and ending points of blink artifact zones. From an obtained peak point of blink signal, a corresponding cell zone can be gotten that satisfies a restriction to the average and variance of squared absolute value of instantaneous frequency. Then expand the zone until the average and variance are exceeded. This zone is preliminarily identified as one of blink artifact zones. After the isolative zones are obtained, we compare all the zones and set a constraint critical value. With the critical value the zones of blink artifacts are identified out.

\section{WAVELET CoEfFICIENTS PROCESSING}

For the processing of blink artifact removal, our basic idea is to remove artifacts and retain the original state of non contaminated parts of the EEG signal, which accord with common knowledge and is consistent with manual processing of blink artifact removal.

We know that the criteria for signal de-noising should be that the processed signal does not lose the key information of an original signal. Usually the wavelet de-noising method is to select a threshold value or different threshold values and a threshold function after the decomposition of a signal to process related coefficients [12]. Then a reconstructed signal is used as a de-noising result.

However, blink artifact removal is different from usual signal de-noising problem. A blink artifact is not white noise mixed on EEG signal. It is typical colored noise and its amplitude of fluctuation is significantly higher than the amplitude of clean EEG signal. It is difficult to obtain reliable result by using usual threshold and threshold function method. In fact the problem has been felt by the researchers [5] when an empirical threshold is gotten. Our method is based on a stationary wavelet transform. The wavelet coefficients are decomposed into the level that coefficients of blink artifact are focused in the approximation coefficients. After that we replace the part of coefficients by polynomial fitting coefficients with the down sampling data from two sides of a blink zone. We know a blink exists at a very short time. If there is no blink appearance at a short period of time, the EEG signal in the corresponding zone should be basically consistent with the change trend of both sides of the zone.

From biomedical conclusion and frequency spectrums of blink artifact zones cut off from EEG signals, we know that the detail coefficients of blink artifact zones come from EEG signal. In addition, detail coefficients include white noise in EEG signal which should be removed. We refer to usual wavelet de-noising method. However in processing the detail coefficients of a blink zone, we use the threshold values of the two sides of a blink zone to estimate the threshold value in the zone at each level, rather than using the threshold value obtained from the zone. The purpose is to avoid the influence of blink artifacts. The method is to estimate a threshold value from 1 second in front of the zone of blink artifact with the formula (9), and then estimate a threshold value from 1 second behind the zone. Then an average value of the two threshold values is used in the zone.

$$
\lambda_{\mathrm{i}}=\sigma_{i} \sqrt{2 \log _{e}\left(N \log _{2} N\right)}
$$

In this formula, $\sigma_{i}=M A D_{\mathrm{j}} / 0.6745, M A D_{\mathrm{j}}$ is an absolute median of wavelet transform coefficients of $\mathrm{j}$ level. 


$$
\hat{\omega}_{\mathrm{j}, k}= \begin{cases}\omega_{j, k} & \left|\omega_{j, k}-\bar{\omega}_{j}\right|<\lambda_{j} \\ \bar{\omega}_{j} & \left|\omega_{j, k}-\bar{\omega}_{j}\right| \geq \lambda_{j}\end{cases}
$$

In this formula, $\bar{\omega}_{\mathrm{j}}$ is a mean value of wavelet transform coefficients at $\mathrm{j}$ level.

The wavelet decomposing coefficients of EEG signals are processed by the piecewise soft threshold function (10). After processing the coefficients, the results of wavelet inverse transform are used to replace related data getting the EEG signal of blink artifact removal and white noise de-noising.

This is an adaptive algorithm on the time scale of wavelet transform. This method is reasonable for the estimation of the amplitude of blink and it does not affect the low frequency component of non blink zones, which retain reasonable EEG signal shape for brain activity study and clinical diagnosis.

\section{EXPERIMENTAL RESULTS}

The experimental results are obtained with above proposed algorithm on the data of visual evoked potential signals. The signals come from Salk computational neurobiology Laboratory of California University in San Diego, hat consist of continuous records of 32 lead EEG data, including 2 lead synchronous eye electrical recording signals. Data recording time is 238 seconds, sampling frequency $128 \mathrm{~Hz}$.

Physiological artifact signals in EEG are often called as interference noise in literatures. These interferences can appear randomly. Although the design of the original experiment is conducive to limit blink and eye movement, the EEG signal is still contaminated by them. The blink artifacts are significant in Fp1, Fpz, Fp2, F3, Fz, F4 lead locations of the electrode records. In our experiment, the signal in Fp1 lead location is used to test and verify the method proposed in this paper. In Fp1 lead location, the interference of blink artifacts to the EEG signal is strong, which is the appropriate target of eliminating the artifacts.

Fig. 3 gives the electrical energy distribution of the EEG signal at the Fp1 electrode position. On the right side of the figure is event related potential (ERP) diagram. Below is the power spectrum of the signal. At the bottom of Fig. 3, SAD refers to the mean difference value of energy distribution space. Now the feature value is significantly more than the threshold, which shows that in the frontal lobe the amplitude of the frontal lobe is significant, compared to the posterior zone. The feature value TK expresses the sensitivity of time to amplitude peak value. Here TK is significantly larger than the threshold, an abnormal amplitude distribution. These feature values give a typical sign of blink appearance.

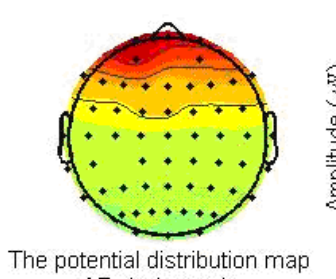

of Fp1 electrode

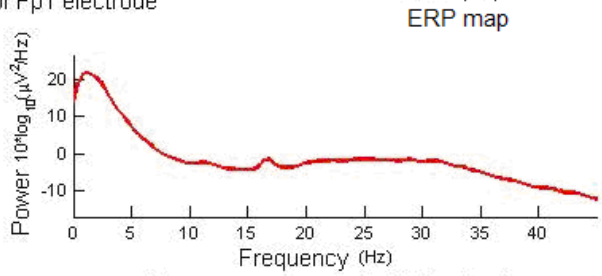

Power spectrum of the EEG signal

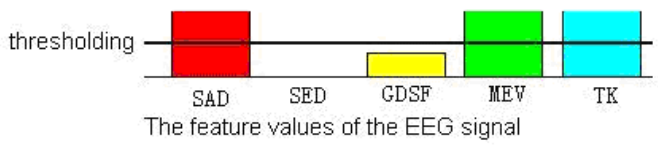

Figure 3. The map of the energy distribution, ERP, power spectrum and feature values in the experiment data.

Taking into account our experimental purpose and program running time, we select the experimental data segment as Fig. 1. Above Fig. 1 comes from one segment of the signal. In the following Fig. 6-7, selected time range is slightly larger than the range in Fig. 1. The length of each data segment is 2048 sampling points. Each segment includes 2-3 blink artifacts. The signals are decomposed by a stationary wavelet transform. The decomposition depth is 5 levels. Fig. 4 (corresponding to Fig. 1) shows the EEG data waveform, in which two blink artifacts appear.

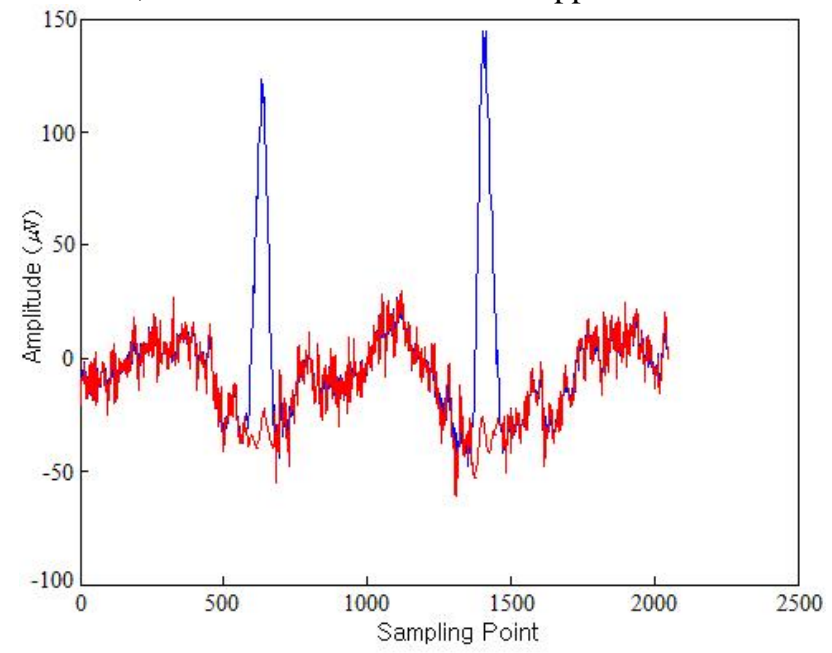

Figure 4. The waveforms of the original EEG signal and the signal of blink artifact removal.

In Fig. 4, the blue wave line indicates the original EEG signal. The red wave line indicates the EEG signal removed blink artifacts, which is attached to the original EEG signal waveform. If there are no blink artifact appearances, we can think that the red wave line is a more credible change trend. We believe that it is appropriate to provide physicians with 
the result that is as far as possible to retain the original signal. On the other hand, the results may be quite consistent with manual processing results. However we introduce the quantitative method for blink artifact removal, which are unavailability by physicians because manual processing is subjective.

Fig. 5 shows the power spectral density (PSD) of the original EEG signal that is contaminated by blink artifacts, the PSD of the signal of blink artifact removal and the PSD of the EEG signal that comes from the data segment of the original EEG signal uncontaminated by blink artifact. Here the last signal is considered as a clean EEG signal. In Fig. 5, the blue wave line indicates the PSD map of the original EEG signal. The green wave line indicates the PSD map of the EEG signal removed blink artifacts. The red wave line indicates the PSD map of the clean EEG signal. We know that the three kinds of signals cannot exist at the same data segment in the same data. But from the result we still can see that the PSD of blink artifact removal signal is closer to the PSD of the clean EEG signal.

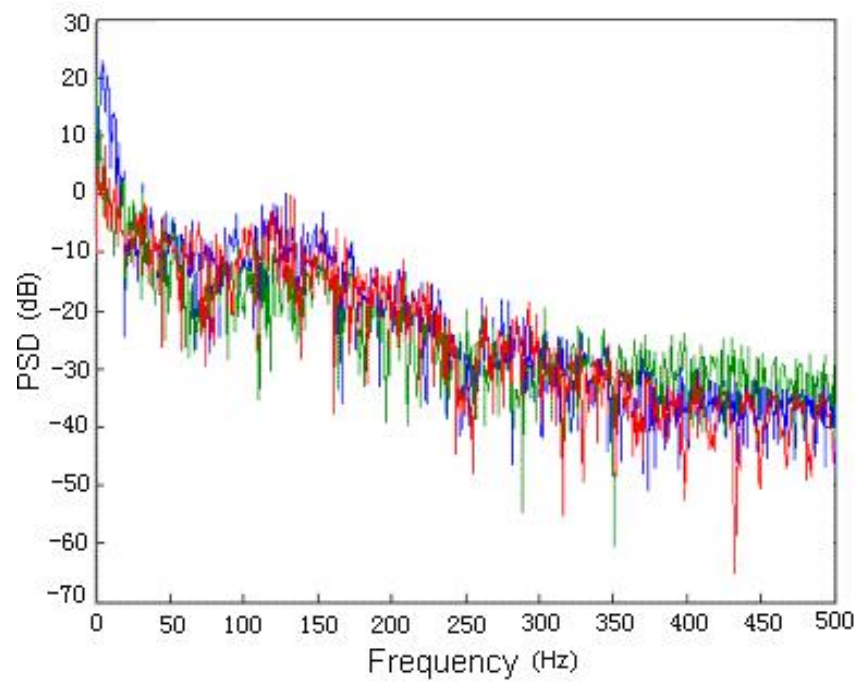

Figure 5. The power spectrum density diagram of the three EEG signals in the experiment.

As a contrast experiment, we used the software, with popular ICA method [13-14], from Salk computational neuroscience laboratory to obtain the result of blink artifact removal. ICA method requires multiple lead signals, which is satisfied by the 32 lead EEG data above mentioned. The result is shown in Fig. 6. As another contrast experiment, we use the software, with blind source separation method, from Finland Tampere University of Technology [15], to remove blink artifacts. The experimental result is shown in Fig. 7. In Fig. 6, the original signal and the signal of blink artifact removal are superimposed together. The blue wave line indicates the original signal and red wave line indicates the signal of blink artifact removal. At the positions $\mathrm{t} 1$ and $\mathrm{t} 2$, we can observe the difference between the original signal and the signal of blink artifact removal. The latter could not conform to the reasonable fluctuation tendency of the signal at the points and the related small zone. In addition, it can be found that the red wave line indicates that the high frequency component of the processed signal is increased, in fact which does not meet the usual requirement of signal de-noising. In Fig. 7 the original signal and the signal of blink artifact removal are up and down reference. At the positions t1 and t2, they can be observed to the result of blink artifact removal losses the fluctuation tendency of the original signal. This result is difficult for physicians to clearly understand the impact of a mathematical method and computer program on the signal. Comparing Fig. 6-7 and Fig. 4, we can see that the proposed method in this paper is effective. The results obtained by our computer program can achieve more reasonable fluctuation tendency of the EEG signal after blink artifact removal.

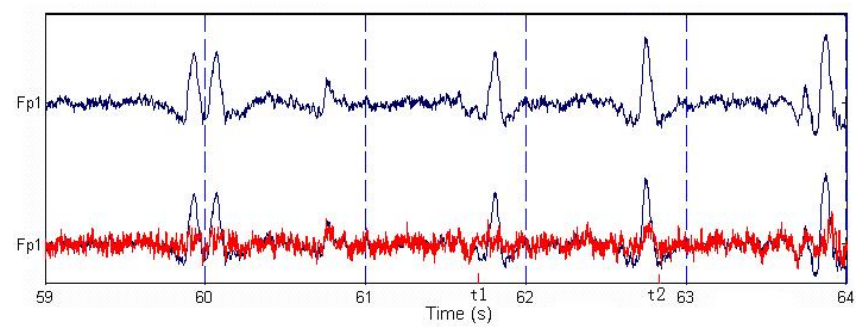

Figure 6. The contrast waveforms of the original EEG signal and the signal of superimposed blink artifact removal with ICA method.

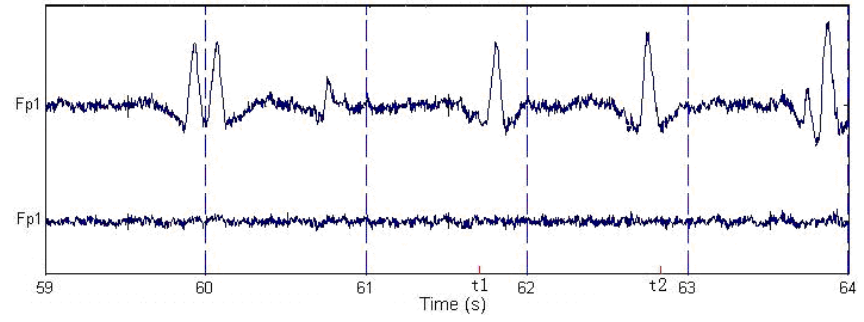

Figure 7. The contrast waveforms of the original EEG signal and the signal of blink artifact removal with blind source separation techniques.

\section{CONCLUSION}

The removal of blink artifact of EEG signal is of considerable importance for both the automated and visual analysis of underlying brainwave activity. Blink artifact increases the difficulty in analyzing the EEG and obtaining clinical information. Due to the limitations of signal acquisition environment and special application needs, single lead EEG signal processing is a question of concern in related research fields. From the signal processing point of view, single lead EEG signal processing does not require the multiple lead signals or the reference signals that are needed by other methods. The method proposed in this paper, for single lead EEG signal processing, is compared with the ICA method and the blind source separation method. The results show that the proposed method is effective.

The method for the removal of blink artifacts does not have an influence on the non blink artifact zones, which accords with usual cognition of the blink artifacts influence on EEG signals and manual processing result of the artifact removal. However this method overcomes the limitation of 
manual processing in quantification and avoids heavy burden of manual processing.

\section{REFERENCES}

[1] Saleha K, Ruhi M, and Bashir I. M., "Comparative Analysis of Wavelet Based Approaches for Reliable Removal of Ocular Artifacts from Single Channel EEG,” 2015 IEEE International Conference on Electro/Information Technology, IEEE Press, 21 May 2015, pp. 335-340, doi: 10.1109/EIT.2015.7293364.

[2] H Anh, T Nguyen, J Musson, etc., "EOG artifact removal using a wavelet neural network,” Neurocomputing vol. 97, Feb. 2012, pp. 374-389, doi: 10.1016/j.neucom.2012.04.016.

[3] V.Krishnaveni1, S.Jayaraman1 , S.Aravind1, etc., "Automatic Identification and Removal of Ocular Artifacts from EEG using Wavelet Transform," Measurement Science Review, vol. 6, July 2006, pp.45-60, oai:CiteSeerX.psu:10.1.1.99.9641.

[4] M Kaiyu, W Ming, Y Hui, etc., "A method for blink artifact removal based on wavelet singularity detection and thresholding denoising," Beijing Biomedical Engineering, vol. 3, June 2015, pp. 251-255.

[5] SP Kumar, R Arumuganathan, K Sivakuma, etc., "Removal of Ocular Artifacts in the EEG through Wavelet Transform without using an EOG Reference Channel,” Int. J. Open Problems Compt. Math., vol. 1, Dec. 2008, pp. 188-200, doi:10.1007/s11277-014-1961-2.

[6] Claudio B, Claudio D. P. and Anna C. etc., "Cortical sources of resting state EEG rhythms are related to brain hypometabolism in subjects with Alzheimer's disease: an EEG-PET study," Neurobiology of Aging, vol. 48, 2016, pp. 122-134. doi:10.1016/j. neurobiolaging.2016.08.021.

[7] Mumtaz H S, Nasreen B, Mohd Z Y, etc., "A Method for Automatic Removal of Eye Blink Artifacts from EEG Based on EMD-ICA," IEEE 9th International Colloquium on Signal Processing and its
Application, 8-10 Mar., 2013, pp. 129-134. doi:10.1109/CSPA.2013. 6530028.

[8] P Venkatakrishnan and S Sangeetha, "Singularity detection in human EEG signal using wavelet leaders,” Biomedical Signal Processing and Control. vol. 13, Sept. 2014, pp. 282-294, doi:10.1016/j.bspc. 2014.06.002.

[9] Zh Hongwei, H Xiangyun, S Wei, etc., "Status Quo of Hilbert Transform in Geophysical Prospecting," Chinese Journal of Engineering Geophysics, vol. 10, July, 2013, pp. 571-575.

[10] Zh Hua,W Xiaokai, G jinghuai, etc., "Local Instantaneous Frequency Extracting Method and its Application to the Daqing Oil Field," Progress in Geophysics (Chinese), vol.26, Jun. 2011, pp, 1064-1069.

[11] W Yingli and W Zhensen, "Micro-vibration Laser Doppler Signal Processing Based on Hilbert Transform,” Chinese Optics, vol. 6, 2013, pp. 415-420.

[12] Hong P, Bin H and Qiuxia S, etc., "Removal of ocular artifacts in EEG--an improved approach combining DWT and ANC for portable applications," IEEE Journal of Biomedical \& Health Informatics, vol. 17, May, 2013, pp. 600-607, doi: 10.1109/JBHI.2013.2253614.

[13] R Grandchamp, C Braboszcz, S Makeig, etc., "Stability of ICA decomposition across within-subject EEG datasets," 34th Annual International Conference of the IEEE EMBS, IEEE Press, 28 Aug. 1 Sep. 2012, pp. 6735-6739, doi: 10.1109/EMBC.2012.6347540.

[14] JR Iversen and S Makeig, "MEG/EEG Data Analysis Using EEGLAB," Magnetoencephalography: From Signals to Dynamic Cortical Networks, Springer Press, May, 2014, pp. 199-212. doi:10. 1007/978-3-642-33045-2.

[15] G Gómezherrero, "Automatic Artifact Removal (AAR) toolbox v1.3 (Release 09.12. 2007) for MATLAB,” Tampere University of Technology Press, Dec. 2007, pp. 1-23. 